\title{
Treatment Initiation for New Episodes of Depression in Primary Care Settings
}

\author{
Beth Waitzfelder, $P h D^{7}$, Christine Stewart, $P h D^{2}$, Karen J. Coleman, $P h D^{3}$, \\ Rebecca Rossom, MD, MS ${ }^{4}$, Brian K. Ahmedani, PhD ${ }^{5}$, Arne Beck, PhD 6 , John E. Zeber, PhD', \\ Yihe G. Daida, $P h D^{7}$, Connie Trinacty, $P h D^{7}$, Samuel Hubley, $P h D^{6}$, and Gregory E. Simon, $M D, M P H^{2}$
}

'Kaiser Permanente Center for Health Research - Hawaii, Honolulu, HI, USA; ${ }^{2}$ Kaiser Permanente Washington Health Research Institute, Seattle, WA, USA; ${ }^{3}$ Department of Research and Evaluation, Kaiser Permanente Southern California, Pasadena, CA, USA; ${ }^{4}$ HealthPartners Institute, Minneapolis, MN, USA; ${ }^{5}$ Center for Health Policy \& Health Services Research, Behavioral Health Services, Henry Ford Health System, Detroit, MI, USA; ${ }^{6}$ Institute for Health Research, Kaiser Permanente Colorado, Denver, CO, USA; ${ }^{7}$ Baylor Scott \& White Health, Center for Applied Health Research, Temple, TX, USA.

BACKGROUND: Depression is prevalent and costly, but despite effective treatments, is often untreated. Recent efforts to improve depression care have focused on primary care settings. Disparities in treatment initiation for depression have been reported, with fewer minority and older individuals starting treatment.

OBJECTIVE: To describe patient characteristics associated with depression treatment initiation and treatment choice (antidepressant medications or psychotherapy) among patients newly diagnosed with depression in primary care settings.

DESIGN: A retrospective observational design was used to analyze electronic health record data.

PATIENTS: A total of 241,251 adults newly diagnosed with depression in primary care settings among five health care systems from 2010 to 2013.

MAIN MEASURES: ICD-9 codes for depression, following a 365-day period with no depression diagnosis or treatment, were used to identify new depression episodes. Treatment initiation was defined as a completed psychotherapy visit or a filled prescription for antidepressant medication within 90 days of diagnosis. Depression severity was measured with Patient Health Questionnaire (PHQ-9) scores on the day of diagnosis.

KEY RESULTS: Overall, $35.7 \%$ of patients with newly diagnosed depression initiated treatment. The odds of treatment initiation among Asians, non-Hispanic blacks, and Hispanics were at least 30\% lower than among nonHispanic whites, controlling for all other variables. The odds of patients aged $\geq 60$ years starting treatment were half those of patients age 44 years and under. Treatment initiation increased with depression severity, but was only $53 \%$ among patients with a PHQ -9 score of $\geq 10$. Among minority patients, psychotherapy was initiated significantly more often than medication.

CONCLUSIONS: Screening for depression in primary care is a positive step towards improving detection, treatment, and outcomes for depression. However, study results indicate that treatment initiation remains suboptimal, and disparities persist. A better understanding of patient

Received March 19, 2017

Revised August 30, 2017

Accepted December 22, 2017

Published online February 8, 2018 factors, and particularly system-level factors, that influence treatment initiation is needed to inform efforts by heath care systems to improve depression treatment engagement and to reduce disparities.

KEY WORDS: depression; primary care; race and ethnicity; disparities.

J Gen Intern Med 33(8):1283-91

DOI: $10.1007 / \mathrm{s} 11606-017-4297-2$

(C) Society of General Internal Medicine 2018

\section{INTRODUCTION}

In the United States, more than 16 million adults (6.7\%) experience an episode of major depression each year. ${ }^{1}$ Depression is among the costliest of public health conditions in the US, with an estimated annual cost of $\$ 210$ billion, due to medical care and lost productivity. ${ }^{2}$ The reported prevalence of depression is higher among women, younger adults, and non-Hispanic whites (NHWs) than among men, older adults, and minority populations. ${ }^{1,3}$ Despite the wide availability of effective treatments for depression, it is estimated that more than half of those with depression do not initiate treatment. ${ }^{2,4}$

Some patient populations appear to be particularly vulnerable to lack of treatment for depression. Studies have reported that treatment initiation among racial and ethnic minority populations is lower than in NHW populations..$^{5-7}$ In addition, among patients who do start treatment, the use of antidepressant medications (AD) is reportedly higher in NHW populations than in minority populations. ${ }^{8-11}$ These differences in treatment engagement and treatment choice likely reflect a combination of patient preference and provider and health system factors, although the precise mechanisms underlying these differences are not well understood.

Over the past decade, there has been increasing focus on the role of primary care in the detection and treatment of depression. Many people with depression, even those who have died by suicide, have never had a mental health diagnosis or received treatment. ${ }^{12,13}$ While behavioral health services may be underutilized, most people do seek primary care; therefore, this 
care setting has been identified as presenting an opportunity to detect and treat depression. The US Preventive Services Task Force (USPSTF) recommendations include depression screening in the general adult population. ${ }^{14}$ The National Committee for Quality Assurance (NCQA) currently includes a measure for $\mathrm{AD}$ adherence and is planning measures for depression screening and follow-up. ${ }^{15,}{ }^{16}$ Efforts to improve the quality of depression treatment in primary care settings include increased use of brief screening tools, such as the Patient Health Questionnaire (PHQ-9), ${ }^{17}$ and implementation of various forms of collaborative and integrated care models. ${ }^{18-20}$ These initiatives have firmly established depression screening and treatment as essential components of primary care. ${ }^{16,21-23}$

This study describes patient characteristics associated with depression treatment initiation and specific treatment choice among a sample of over 240,000 patients who received a new diagnosis of depression in primary care settings across five large, integrated health care systems between 2010 and 2013. These health care systems are members of the Mental Health Research Network (MHRN), a consortium of 13 health care systems providing primary and specialty care to a combined patient population of over 12 million. ${ }^{24}$ Based on a large and diverse patient population, this study describes current depression treatment initiation patterns among multiple US health care systems striving to enhance depression care in primary care settings in response to national initiatives.

\section{METHODS}

\section{Setting: Health Care Systems}

Study data were obtained from five US health care systems with diversity in size, geographic location, and patient populations: Kaiser Permanente regions of Southern California, Washington, Colorado, and Hawaii, and HealthPartners in Minnesota. At the time of analysis, these systems provided both private, primarily commercial, and subsidized public insurance coverage and health care to over 5.1 million people in five states. Electronic health records (EHR), insurance claims, and other data are organized in a virtual data warehouse (VDW) to facilitate population-based research across all systems. ${ }^{24,}{ }^{25}$ Protected health information remains at each site, but the VDW uses common data definitions and formats to ensure equivalent de-identified data for analysis. The institutional review boards at each health care system approved this study.

During the study period (2010-2013), these health care systems were using the Patient Health Questionnaire (PHQ9), a brief depression screening tool, ${ }^{17}$ in some capacity in primary care settings. All five systems had implemented evidence-based guidelines for diagnosis and treatment of depression in primary and specialty care clinics, four systems were monitoring and reporting NCQA measures of depression care quality, three systems were following recommendations for the use of standard outcomes assessments for patients receiving depression treatment, and three systems had integrated mental health providers in primary care clinics, during all or part of the study period.

\section{Study Population}

The study included adult patients (age $\geq 18$ ) with a new diagnosis of depression made in a primary care setting in one of the five study sites between 2010 and $2013(N=241,251)$. Using VDW information, new episodes of depression were defined by the presence of an ICD-9 code for depression following a 365-day period without evidence of a depression diagnosis or treatment (either psychotherapy or AD). Cases were followed for 90 days after the diagnosis (index) date to look for the initiation of AD medication or psychotherapy treatment. PHQ9 scores measured on the index date were available for 27,347 patients, $11 \%$ of the sample. Patients who were disenrolled from the health system within 90 days after diagnosis were excluded $(n=6207,2.6 \%)$.

\section{Measures}

VDW data was the source for demographic variables, including age, gender, and race/ethnicity. The Charlson index was used as a comorbidity index, using diagnosis codes appearing in the EHR during the year prior to the index date. ${ }^{26}$ Neighborhood income and education were imputed using geocoded patient addresses and census data at the block group level. VDW data were assessed for evidence of prior AD use, the receipt of mental health specialty care, and prior hospitalization with a mental health diagnosis during the 5-year period prior to the index date. Treatment initiation was measured as a filled prescription for any $\mathrm{AD}$ or at least one completed psychotherapy visit within 90 days after the index date. A psychotherapy visit was defined as any visit greater than 30min duration to a specialty mental health provider with a Current Procedural Terminology (CPT) code indicating either initial evaluation or individual psychotherapy. Detailed specifications for antidepressant prescription fills and psychotherapy visits are available at: https://github.com/MHResearchNetwork/MHRN-Central (MHRN_psychotherapyList.xls, mhrn2_ndc2016a.zip). Where available, PHQ-9 scores were used in analyses as a dichotomous measure of depression severity, with a score of $\geq 10$ indicative of probable major depression. ${ }^{17}$

\section{Analyses}

Logistic regression models were first used to estimate the odds of initiating treatment ( $\mathrm{AD}$ and/or psychotherapy) by patient characteristics. Among the subgroup of patients who did initiate treatment, logistic regression models were then used to estimate the odds of initiating psychotherapy (as opposed to AD) by patient characteristics. Patients who initiated both psychotherapy and $\mathrm{AD}$ were included in the models for treatment initiation but were excluded from the models for type of treatment initiated. All models included a variable for health 
care system. Wald tests were used to calculate the $p$ values for the association between each variable and model outcomes (treatment initiated or not, and psychotherapy [vs. AD] initiated). The models were then used for analyses of the subgroup of patients who had PHQ-9 scores on the index date. All analyses were conducted using SAS version 9.4 software. ${ }^{27}$

\section{RESULTS}

\section{Patient Characteristics}

Of the 241,251 patients who received a new diagnosis of depression, $48 \%$ were NHW, 26\% were Hispanic, 7\% were non-Hispanic black, and 5\% were Asian. Nearly 69\% were female, and about $66 \%$ were under the age of 60 (Table 1). The subgroup with a PHQ-9 score recorded at the time of diagnosis included 27,347 patients (11\%). This screened group had a larger proportion of NHW (62\%) and younger patients (73\% under age 60). Race/ethnicity was unknown or "other" for $12 \%$ of the cohort and 5\% of the subgroup with PHQ-9 scores.

\section{Initiation of Treatment}

Overall, of the 241,251 new episodes of diagnosed depression during this period, $86,115(35.7 \%)$ initiated $\mathrm{AD}$ medication and/or psychotherapy (Table 1). Age, race/ethnicity, and health care system were the strongest predictors of treatment initiation. The adjusted odds ratio (aOR) for starting treatment declined with increasing age, with the aOR for those aged 60 and older less than half that for those age 44 years and under. In comparison to NHWs, all racial and ethnic minority groups had significantly lower odds of starting treatment: aORs ranged from 0.65 for Asians to 0.83 for Native Americans, with nonHispanic blacks, Hispanics, and Native Hawaiians/Pacific Islanders in the $0.67-0.72$ range. Men had slightly higher odds than women (aOR 1.07, CI 1.05-1.09). The aORs for starting treatment ranged from 0.66 to 1.03 across the five study sites.

Increasing levels of clinical comorbidity were associated with higher odds of treatment initiation. Prior use of specialty mental health care (aOR 0.86, CI 0.84-0.88) and prior hospitalization with a mental health diagnosis (aOR 0.78 , CI $0.71-$ 0.78 ) were associated with lower odds of starting treatment for this new depression episode. Prior AD use was not associated with starting treatment. Higher education and income levels were associated with slightly increased odds of starting treatment.

Results for the 27,347 cases with PHQ-9 scores simultaneously recorded on the index date are shown in Table 1 . Overall, $45 \%$ of these patients initiated treatment. In this model, PHQ-9 score, study site, race/ethnicity and age were the strongest predictors of treatment initiation. As expected, the odds of patients with high PHQ-9 scores $(\geq 10)$ starting treatment were 3.34 times higher than those with low PHQ-9 scores. Fifty-three percent of patients with PHQ-9 scores $\geq 10$ initiated treatment. Similar to the total cohort, the aORs for treatment initiation among this subsample of patients were lower for racial/ethnic minority groups than for NHWs. The lowest odds were among Hispanics (0.60, CI 0.56-0.65), Asians (0.65, CI 0.58-0.72), and non-Hispanic blacks (0.67 CI 0.61-0.75). The odds of initiating treatment again decreased with increasing age, but the differences were smaller in this subsample of patients. Adjusted odds ratios of treatment initiation ranged from 0.80 to 1.98 across the five study sites.

\section{Initiation of Psychotherapy Compared to Antidepressant Medications}

More than $80 \%$ of patients who initiated treatment started an AD. As shown in Table 2, the strongest predictors of initiating psychotherapy rather than medication were age, race/ethnicity, prior $\mathrm{AD}$ use, and health care system. Prior use of mental health specialty care, fewer medical comorbidities, and male sex were associated with increased odds of initiating psychotherapy.

The proportion of patients initiating psychotherapy decreased with increasing age, with $25 \%$ of 18-29-year-olds starting psychotherapy compared to $7 \%$ of patients aged 75 and older. While fewer patients of racial minorities initiated treatment, among those who did, all racial and ethnic minority groups had higher proportions of psychotherapy initiation than NHWs. Thirty percent of non-Hispanic black patients started psychotherapy, in comparison to $14 \%$ of NHWs, $24 \%$ of Hispanics, 24\% of Asians, and 21\% of Native Hawaiians/ Pacific Islanders. Men had higher odds of starting psychotherapy than women.

Prior use of $\mathrm{AD}$ medications was associated with lower odds of starting psychotherapy (aOR 0.46, CI 0.44-0.49), but patients who had prior use of specialty mental health care had higher odds of starting psychotherapy. The aORs for initiating psychotherapy varied from 0.54 to 1.39 across the five study sites.

Results for the 9871 patients who initiated treatment and also had a PHQ-9 score are shown in Table 2. Health care system, previous use of $\mathrm{AD}$ medications, race/ethnicity and age were the strongest predictors of psychotherapy initiation. The aORs for psychotherapy initiation varied from 0.35 to 1.94 across study sites. The aOR of initiating psychotherapy was 0.39 (CI 0.34-0.45) among patients who had used AD medications in the past. The aOR for psychotherapy initiation remained higher for all racial/ethnic groups in comparison to NHWs. Initiation of psychotherapy decreased with increasing age. Patients with high PHQ scores $(\geq 10)$ had $30 \%$ lower odds of initiating psychotherapy than patients with low PHQ scores. The odds of starting psychotherapy among men in this group were $23 \%$ higher than those for women.

\section{DISCUSSION}

The results of this study highlight persistent suboptimal levels of treatment initiation for depression, as well as age and racial/ 


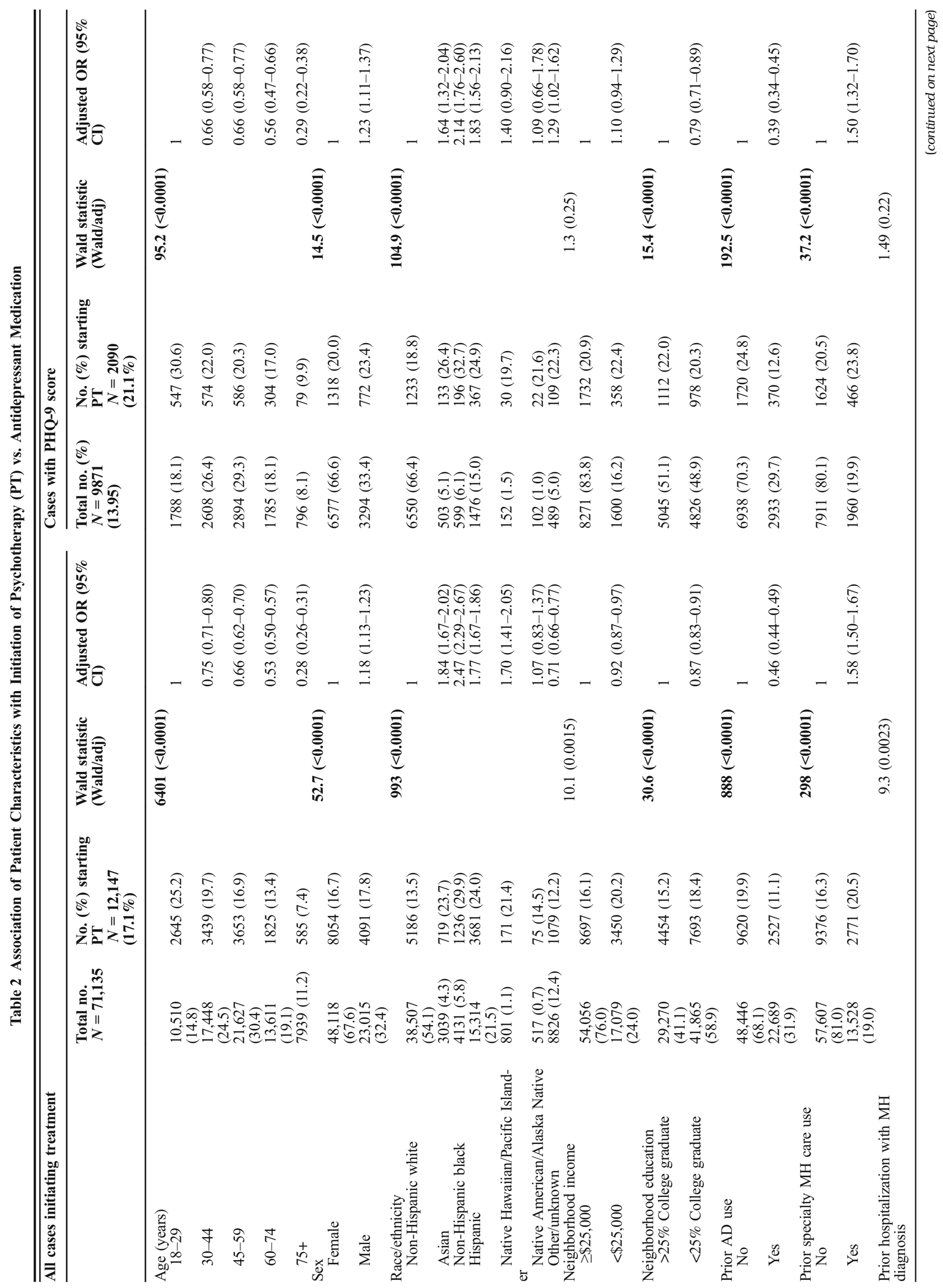




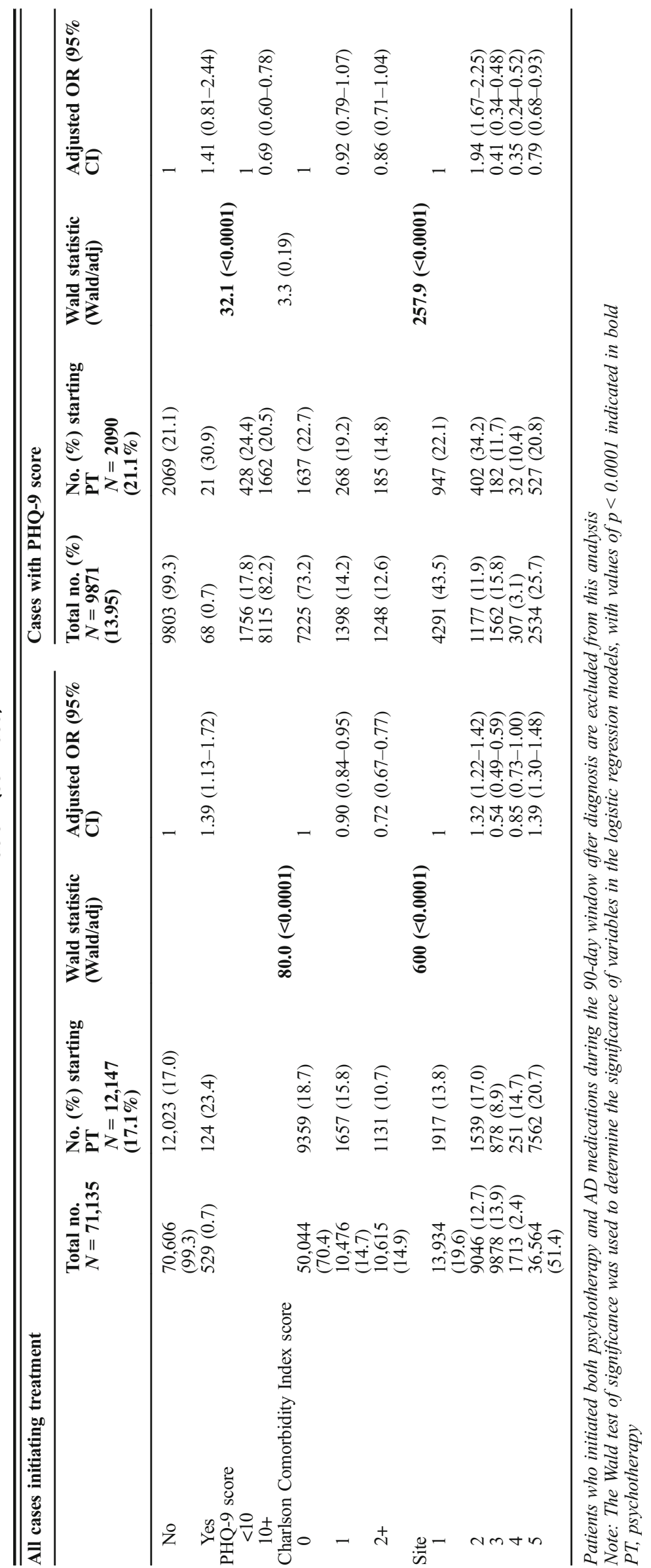


ethnic disparities in the initiation of treatment, despite initiatives to enhance depression care in primary care. The study also highlights variations among health care systems in the initiation of treatment for depression, underscoring the importance of better understanding the effectiveness of depression care integration mechanisms and processes. Importantly, the study results reflect recent practice patterns among multiple health care systems, and are based on a significantly larger $(n=241,251)$ and more diverse patient population than those included in previous studies. The results are strengthened by the inclusion of important covariates (e.g., socioeconomic indicators, prior mental health service use) by leveraging uniform EHR data.

\section{Depression Treatment Initiation in Primary Care}

The observed proportion of patients initiating treatment for new episodes of depression diagnosed in primary care was low-36\%. This finding is consistent with previous reports. ${ }^{28}$

${ }^{31}$ Reasons cited in previous studies for low levels of treatment initiation - stigma, patient resistance, insufficient training or discomfort of primary care providers, and access barriers, particularly for behavioral health services - likely contributed to the low levels of treatment initiation observed in this study. ${ }^{32,33}$

Barriers to treatment initiation specific to primary care settings were also likely contributors. These include greater aversion to depression treatment among primary care patients than behavioral health patients, competing demands, time constraints, and different priorities for patients and providers. $^{34,35}$

\section{Patient Characteristics, Treatment Initiation, and Treatment Choice}

Race/Ethnicity. The results demonstrate significant, persistent differences in depression treatment initiation associated with race/ethnicity. The odds of Asians, non-Hispanic blacks, and Hispanics initiating treatment were at least $30 \%$ lower than those for NHWs, after controlling for all other variables. This finding is consistent with previous evidence of racial and ethnic disparities in depression treatment initiation. ${ }^{6,7,9,36}$

This study also provides evidence of a preference among minority patients for psychotherapy over AD treatment. ${ }^{8-11}$ Access to one's preferred choice of depression treatment has been found to enhance treatment initiation, adherence, and outcomes. $^{37,38}$

Age. Persistent significant age-related differences were found in treatment initiation. The odds of patients aged 60 years and older starting treatment were half those of patients age 44 years and under. With a rapidly growing aging population, the importance of addressing the mental health needs of this group will increase. Previously reported depression treatment gaps for older patients are further supported by this study. ${ }^{39,40}$
Lower treatment initiation among older patients has been attributed to a common misconception of depression as a natural part of the aging process, a generational culture of personal responsibility, attribution of depression to nonmedical causes, and stigma. ${ }^{39,41,42}$ Resistance to AD treatment has also been identified in this population. ${ }^{43}$

Comorbid medical conditions among older patients may compete for the attention of primary care providers and potentially mask or overlap with depression symptoms. ${ }^{39}$ The results of this study show slightly higher odds of initiating treatment as comorbidities increase. Improved medical disease and depression outcomes have been reported with collaborative care approaches for patients with depression and common comorbidities, particularly diabetes and cardiovascular disease. $^{18,44-46}$

Gender. Although depression is more common among women than men $(8.2 \%$ vs. $4.6 \%),{ }^{1}$ the study results revealed a slightly higher proportion of men initiating treatment. In addition, the odds of starting psychotherapy were $18 \%$ higher for men than for women. These gender-related differences in treatment initiation are a particularly important and positive finding, given that men account for more than threequarters of suicides among middle-aged adults. ${ }^{47}$

Among those who started treatment, psychotherapy was initiated by $17 \%$, and $83 \%$ started $\mathrm{AD}$ medications. This high proportion of $\mathrm{AD}$ use may reflect the large proportion of NHWs in the study population (47\%), greater familiarity with $\mathrm{AD}$ among primary care providers, a desire to rapidly address a newly identified condition, and possibly access barriers to behavioral health specialty care.

\section{Health Care System Factors}

Large differences in treatment initiation were observed across the five participating health care systems, with aORs ranging from 0.66 to 1.03 . While all sites had taken steps to enhance depression care, including the use of the PHQ-9 depression scale in some manner in primary care, the specific features and full scope of efforts to improve the quality of depression care in primary care varied across sites and within sites over time. For example, mechanisms for psychotherapy referral might have ranged from an instant consultation with a behavioral health specialist co-located in primary care at the time of the visit, to simply giving the patient a phone number. While it was not possible in this study to retrospectively reconstruct patient-level exposure to health care system initiatives to enhance depression care, the study results highlight the importance of doing so in the future. The proportion of new diagnoses with a concurrent PHQ-9 score (an important feature of care integration) ranged from 5\% to $33 \%$ across sites (not shown). Higher treatment initiation among the screened group of patients could reflect a more focused approach to screening versus a general screening approach. 


\section{Limitations}

Study limitations include the omission of any brief counseling provided by primary care physicians upon diagnosis. An important limitation is that we have little information about the reasons for failure to initiate treatment, or the relative contribution of patient, provider, and system factors. In addition, since depression is common in the study population, odds ratios may slightly overestimate the associations with predictive factors. ${ }^{48}$ Finally, while all study sites were using the PHQ-9, this study lacks detailed information about the specific conditions under which the tool was utilized, and particularly methods of care integration at the study sites during the study period.

\section{Next Steps}

Efforts to integrate behavioral health care within primary care settings have been under way and evolving across the United States for more than 15 years. The features of collaborative care models vary widely, but there is evidence that these models can be effective in improving depression management and outcomes ${ }^{19,20,49,50}$ and that they are cost-effective. ${ }^{51-53}$ There is also evidence that collaborative care models can be effective for particular patient groups, including younger ${ }^{54}$ and older populations ${ }^{51,55-57}$ and racial minorities. ${ }^{58-62}$ Some models of care have been reported to reduce racial/ethnic disparities. ${ }^{63}$ While these models may not be universally effective, ${ }^{62,64,65}$ regulatory requirements and the desire to better meet patients' mental health care needs will lead to further implementation efforts.

The results of this study provide evidence that a substantial number of patients with diagnosed depression do not receive treatment, even in leading health care organizations. Ongoing efforts to address this problem, coupled with more thorough and sophisticated evaluation methods, will enhance our understanding of the mechanisms by which various models succeed in improving treatment engagement, incorporating patient preferences, improving adherence and outcomes, and reducing disparities.

Acknowledgements: This study was supported by NIMH Cooperative Agreement U19MH092201.

Corresponding Author: Beth Waitzfelder, PhD; Kaiser Permanente Center for Health Research - Hawaii, Honolulu, HI, USA (e-mail: Beth. E.Waitzfelder@kp.org).

\section{Compliance with Ethical Standards:}

Conflict of Interest: Dr. Simon receives royalties from Wolters Kluwer for editing chapters of the UpToDate decision support system. In the last 3 years, he has received research grant support from Novartis Pharmaceuticals for research regarding suicidal behavior in psoriasis. Dr. Daida receives research grant support from Gilead Sciences and Intercept Pharmaceuticals for research regarding hepatitis and fibrotic liver diseases, respectively. Research funds are received through the Henry Ford Health System (prime site for both studies).

All other authors declare that they do not have a conflict of interest.

\section{REFERENCES}

1. National Institute of Mental Health: Major Depression Among Adults. https://www.nimh.nih.gov/health/statistics/prevalence/major-depression-among-adults.shtml. Accessed 21 Dec 2017.

2. National Network of Depression Centers: Facts. http://www.nndc.org/ the-facts/. Accessed 21 Dec 2017.

3. Coleman KJ, Stewart C, Waitzfelder BE, et al. Racial-Ethnic Differences in Psychiatric Diagnoses and Treatment Across 11 Health Care Systems in the Mental Health Research Network. Psychiatric Serv (Washington, DC). 2016;67(7):749-757.

4. Simon GE. Evidence review: efficacy and effectiveness of antidepressant treatment in primary care. Gen Hosp Psychiatry 2002;24(4):213-224.

5. Sclar DA, Robison LM, Schmidt JM, Bowen KA, Castillo LV, Oganov AM. Diagnosis of depression and use of antidepressant pharmacotherapy among adults in the United States: does a disparity persist by ethnicity/ race? Clin Drug Investig 2012;32(2):139-144.

6. Alegria $\mathbf{M}$, Chatterji $\mathbf{P}$, Wells $\mathbf{K}$, et al. Disparity in depression treatment among racial and ethnic minority populations in the United States. Psychiatric Serv (Washington, DC). 2008;59(11):1264-1272.

7. Virnig B, Huang Z, Lurie N, Musgrave D, McBean AM, Dowd B. Does Medicare managed care provide equal treatment for mental illness across races? Arch Gen Psychiatry 2004;61(2):201-205.

8. Jung K, Lim D, Shi Y. Racial-ethnic disparities in use of antidepressants in private coverage: implications for the Affordable Care Act. Psychiatr Serv (Washington, DC). 2014;65(9):1140-1146.

9. Miranda J, Cooper LA. Disparities in care for depression among primary care patients. J Gen Intern Med 2004;19(2):120-126.

10. Quinones AR, Thielke SM, Beaver KA, Trivedi RB, Williams EC, Fan VS. Racial and ethnic differences in receipt of antidepressants and psychotherapy by veterans with chronic depression. Psychiatr Serv (Washington, DC). 2014;65(2): 193-200.

11. Givens JL, Houston TK, Van Voorhees BW, Ford DE, Cooper LA. Ethnicity and preferences for depression treatment. Gen Hosp Psychiatry 2007:29(3):182-191.

12. Ahmedani BK, Simon GE, Stewart C, et al. Health care contacts in the year before suicide death. J Gen Intern Med 2014;29(6):870-877.

13. Ahmedani BK, Stewart C, Simon GE, et al. Racial/Ethnic differences in health care visits made before suicide attempt across the United States. Med Care 2015;53(5):430-435.

14. U.S. Preventive Services Task Force. Screening for depression: recommendations and rationale. Ann Intern Med. 2002;136(10):760-764.

15. NCQA HEDIS Depression Measures Specified for Electronic Clinical Data Systems. http://www.ncqa.org/hedis-quality-measurement/hedis-learningcollaborative/hedis-depression-measures. Accessed 21 Dec 2017.

16. Anderson B. HEDIS antidepressant medication management measures and performance-based measures: an opportunity for improvement in depression care. Am J Manag Care 2007;13(4 Suppl):S98-102.

17. Kroenke K, Spitzer RL, Williams JB. The PHQ-9: validity of a brief depression severity measure. J Gen Intern Med 2001;16(9):606-613.

18. Katon WJ, Lin EH, Von Korff M, et al. Collaborative care for patients with depression and chronic illnesses. N Engl J Med 2010;363(27):2611-2620.

19. Gilbody S, Bower P, Fletcher J, Richards D, Sutton AJ. Collaborative care for depression: a cumulative meta-analysis and review of longerterm outcomes. Arch Intern Med 2006; 166(21):2314-2321.

20. Hedrick SC, Chaney EF, Felker B, et al. Effectiveness of collaborative care depression treatment in Veterans' Affairs primary care. J Gen Intern Med 2003;18(1):9-16.

21. Siu AL, Bibbins-Domingo $\mathbf{K}$, Grossman DC, et al. Screening for Depression in Adults: US Preventive Services Task Force Recommendation Statement. JAMA 2016;315(4):380-387.

22. Robinson RL, Long SR, Chang S, et al. Higher costs and therapeutic factors associated with adherence to NCQA HEDIS antidepressant medication management measures: analysis of administrative claims. $J$ Manag Care Pharm 2006;12(1):43-54.

23. Pincus HA, Houtsinger JK, Bachman J, Keyser D. Depression in primary care: bringing behavioral health care into the mainstream. Health Aff (Project Hope) 2005;24(1):271-276.

24. Mental Health Research Network. http://hcsrn.org/mhrn/en/. Accessed 21 Dec 2017.

25. Ross TR, Ng D, Brown JS, et al. The HMO Research Network Virtual Data Warehouse: A Public Data Model to Support Collaboration. EGEMS (Washington, DC). 2014;2(1): 1049.

26. Charlson ME, Pompei P, Ales KL, MacKenzie CR. A new method of classifying prognostic comorbidity in longitudinal studies: development and validation. $J$ Chronic Dis 1987;40(5):373-383. 
27. SAS Institute Inc., SAS Software 9.4, Cary, N.C.

28. Thornicroft G, Chatterji S, Evans-Lacko S, et al. Undertreatment of people with major depressive disorder in 21 countries. Br J Psychiatry 2016.

29. Hirschfeld RM, Keller MB, Panico S, et al. The National Depressive and Manic-Depressive Association consensus statement on the undertreatment of depression. JAMA 1997;277(4):333-340.

30. Oquendo MA, Malone KM, Ellis SP, Sackeim HA, Mann JJ. Inadequacy of antidepressant treatment for patients with major depression who are at risk for suicidal behavior. Am J Psychiatry 1999;156(2): 190-194.

31. Pence BW, O'Donnell JK, Gaynes BN. The depression treatment cascade in primary care: a public health perspective. Curr Psychiatry Rep 2012;14(4):328-335.

32. Campbell DG, Bonner LM, Bolkan CR, et al. Stigma Predicts Treatment Preferences and Care Engagement Among Veterans Affairs Primary Care Patients with Depression. Annals Behav Med 2016;50(4):533-544.

33. Nutting PA, Rost K, Dickinson $\mathbf{M}$, et al. Barriers to initiating depression treatment in primary care practice. J Gen Intern Med 2002;17(2):103-111.

34. Klinkman MS. Competing demands in psychosocial care. A model for the identification and treatment of depressive disorders in primary care. Gen Hosp Psychiatry 1997;19(2):98-111.

35. Van Voorhees BW, Cooper LA, Rost KM, et al. Primary care patients with depression are less accepting of treatment than those seen by mental health specialists. J Gen Intern Med 2003;18(12):991-1000.

36. Creedon TB, Cook BL. Access To Mental Health Care Increased But Not For Substance Use, While Disparities Remain. Health Aff (Project Hope) 2016;35(6):1017-1021.

37. Raue PJ, Schulberg HC, Heo M, Klimstra S, Bruce ML. Patients depression treatment preferences and initiation, adherence, and outcome: a randomized primary care study. Psychiatr Serv (Washington, DC). 2009;60(3):337-343.

38. Lin P, Campbell DG, Chaney EF, et al. The influence of patient preference on depression treatment in primary care. Annals Behav Med 2005;30(2):164-173.

39. Charney DS, Reynolds CF, 3rd, Lewis L, et al. Depression and Bipolar Support Alliance consensus statement on the unmet needs in diagnosis and treatment of mood disorders in late life. Arch Gen Psychiatry 2003;60(7):664-672.

40. Lebowitz BD, Pearson JL, Schneider LS, et al. Diagnosis and treatment of depression in late life. Consensus statement update. JAMA 1997;278(14):1186-1190.

41. Switzer JF, Wittink MN, Karsch BB, Barg FK. "Pull yourself up by your bootstraps": a response to depression in older adults. Qual Health Res 2006; 16(9):1207-1216

42. Wittink MN, Givens JL, Knott KA, Coyne JC, Barg FK. Negotiating depression treatment with older adults: primary care providers' perspectives. J Ment Health (Abingdon, England). 2011;20(5):429-437.

43. Givens JL, Datto CJ, Ruckdeschel K, et al. Older patients' aversion to antidepressants. A qualitative study. J Gen Intern Med 2006;21(2):146151.

44. Coventry $\mathbf{P}$, Lovell $\mathbf{K}$, Dickens $\mathbf{C}$, et al. Integrated primary care for patients with mental and physical multimorbidity: cluster randomised controlled trial of collaborative care for patients with depression comorbid with diabetes or cardiovascular disease. BMJ (Clin Res ed). 2015;350:h638.

45. Huffman JC, Mastromauro CA, Beach SR, et al. Collaborative care for depression and anxiety disorders in patients with recent cardiac events: the Management of Sadness and Anxiety in Cardiology (MOSAIC) randomized clinical trial. JAMA Intern Med 2014;174(6):927-935.

46. Stewart JC, Perkins AJ, Callahan CM. Effect of collaborative care for depression on risk of cardiovascular events: data from the IMPACT randomized controlled trial. Psychosom Med 2014;76(1):29-37.
47. Suicide among adults aged 35-64 years-United States, 1999-2010. MMWR. 2013;62(17):321-325.

48. McNutt LA, Wu C, Xue X, Hafner JP. Estimating the relative risk in cohort studies and clinical trials of common outcomes. Am J Epidemiol 2003;157(10):940-943.

49. Garrison GM, Angstman KB, O'Connor SS, Williams MD, Lineberry Tw. Time to Remission for Depression with Collaborative Care Management (CCM) in Primary Care. J Am Board Fam Med 2016;29(1):10-17.

50. Archer J, Bower P, Gilbody S, et al. Collaborative care for depression and anxiety problems. Cochrane Database Syst Rev. 2012;10:Cd006525.

51. Unutzer J, Katon WJ, Fan MY, et al. Long-term cost effects of collaborative care for late-life depression. Am J Manag Care 2008;14(2):95-100.

52. Liu CF, Hedrick SC, Chaney EF, et al. Cost-effectiveness of collaborative care for depression in a primary care veteran population. Psychiatr Serv (Washington, DC). 2003;54(5):698-704.

53. Simon GE, Von Korff M, Ludman EJ, et al. Cost-effectiveness of a program to prevent depression relapse in primary care. Med Care 2002;40(10):941-950.

54. Richardson LP, Ludman E, McCauley E, et al. Collaborative care for adolescents with depression in primary care: a randomized clinical trial. JAMA 2014;312(8):809-816.

55. Chang-Quan H, Bi-Rong D, Zhen-Chan L, Yuan Z, Yu-Sheng P, QingXiu L. Collaborative care interventions for depression in the elderly: a systematic review of randomized controlled trials. $J$ Investig Med 2009;57(2):446-455.

56. Unutzer J, Katon W, Callahan CM, et al. Collaborative care management of late-life depression in the primary care setting: a randomized controlled trial. JAMA 2002;288(22):2836-2845.

57. Hegel MT, Unutzer $\mathbf{J}$, Tang $\mathbf{L}$, et al. Impact of comorbid panic and posttraumatic stress disorder on outcomes of collaborative care for late-life depression in primary care. Am J Geriatric Psychiatry 2005;13(1):48-58.

58. Bauer AM, Azzone V, Alexander L, Goldman HH, Unutzer J, Frank RG. Are patient characteristics associated with quality of depression care and outcomes in collaborative care programs for depression? Gen Hosp Psychiatry 2012;34(1):1-8.

59. Ell K, Katon W, Xie B, et al. Collaborative care management of major depression among low-income, predominantly Hispanic subjects with diabetes: a randomized controlled trial. Diabetes Care 2010;33(4):706713.

60. Ratzliff AD, Ni K, Chan YF, Park M, Unutzer J. A collaborative care approach to depression treatment for Asian Americans. Psychiatr Serv (Washington, DC). 2013;64(5):487-490.

61. Ayalon L, Arean PA, Linkins $\mathbf{K}$, Lynch M, Estes CL. Integration of mental health services into primary care overcomes ethnic disparities in access to mental health services between black and white elderly. Am J Geriatr Psychiatry 2007;15(10):906-912.

62. Arean PA, Ayalon L, Jin C, et al. Integrated specialty mental health care among older minorities improves access but not outcomes: results of the PRISMe study. Int J Geriatr Psychiatry 2008;23(10):1086-1092.

63. Angstman KB, Phelan S, Myszkowski MR, et al. Minority Primary Care Patients With Depression: Outcome Disparities Improve With Collaborative Care Management. Med Care 2015;53(1):32-37.

64. Callahan CM, Hendrie HC, Dittus RS, Brater DC, Hui SL, Tierney WM. Improving treatment of late life depression in primary care: a randomized clinical trial. J Am Geriatr Soc 1994;42(8):839-846.

65. Solberg LI, Crain AL, Jaeckels N, et al. The DIAMOND initiative: implementing collaborative care for depression in 75 primary care clinics. Implement Sci 2013;8:135. 